\title{
Scalp reconstruction: A 10-year experience
}

\author{
Hyeon Uk Jang, \\ Young Woong Choi \\ Department of Plastic and \\ Reconstructive Surgery, Inje University \\ Sanggye Paik Hospital, Inje University \\ School of Medicine, Seoul, Korea
}

\begin{abstract}
Background: The scalp is an important functional and aesthetic structure that protects the cranial bone. Due to its inelastic characteristics, soft-tissue defects of the scalp make reconstruction surgery difficult. This study aims to provide an improved scalp reconstruction decision making algorithm for surgeons.

Methods: This study examined patients who underwent scalp reconstruction within the last 10 years. The study evaluated several factors that surgeons use to select a given reconstruction method such as etiology, defect location, size, depth, and complications. An algorithmic approach was then suggested based on an analysis of these factors.

Results: Ninety-four patients were selected in total and 98 cases, including revision surgery, were performed for scalp reconstruction. Scalp reconstruction was performed by primary closure $(36.73 \%)$, skin graft $(27.55 \%)$, local flap $(17.34 \%)$, pedicled regional flap $(15.30 \%)$, and free flap $(3.06 \%)$. The ratio of primary closure to more complex procedure on loose scalps $(51.11 \%)$ was significantly higher than on tight scalps $(24.52 \%)(p=0.011)$. The choice of scalp reconstruction method was affected significantly by the defect size $(R=0.479, p<0.001)$ and depth $(p<0.001)$. There were five major complications which were three cases of flap necrosis and two cases of skin necrosis. Hematoma was the most common of the 29 minor complications reported, followed by skin necrosis.

Conclusion: There are multiple factors affecting the choice of scalp reconstruction method. We suggest an algorithm based on 10 years of experience that will help surgeons establish successful surgical management for their patients.
\end{abstract}

Keywords: Radiotherapy / Reconstructive surgical procedures / Scalp

\section{INTRODUCTION}

The scalp is an important functional structure that covers the cranial bones and protects the brain from external factors [1]. It is also an important aesthetic structure in that it is hair-bearing. Damage to this structure, such as from trauma, burns, radiation, or tumor excision can result in poor quality of life from

\footnotetext{
Correspondence: Young Woong Choi

Department of Plastic and Reconstructive Surgery, Inje University Sanggye Paik Hospital, Inje University School of Medicine, 1342 Dongil-ro, Nowon-gu, Seoul 01757, Korea

E-mail: pshero@gmail.com

This work was supported by the 2019 Inje University research grant.

Received June 19, 2020 / Revised July 17, 2020 / Accepted August 14, 2020
}

factors such as alopecia or deformity.

The complex anatomy of the scalp makes reconstruction difficult. The scalp consists of tight and loose portions, which surgeons must consider when choosing a method of scalp reconstruction [1]. Additionally, surgeons should consider how the scalp reconstruction can be achieved so as to not distort the hairline and, if possible, minimize scars, alopecia, or deformity [2]. Previous scars or fibrosis may adversely affect the reconstruction for a local or regional flap [3]. Surgeons should choose the best reconstructive procedure for each patient considering factors like defect size, depth, location, hairline, and alopecia risk. Therefore, it is necessary to build a useful manual to more easily choose the best reconstructive method for each 
situation.

The purpose of this study is to compare and analyze the cases of scalp reconstruction that have been performed in a single center over the past 10 years. Using this data, we aimed to provide better surgical choices for scalp reconstructions to surgeons, through the compilation of an algorithm.

\section{METHODS}

This study was a retrospective clinical review of patients who underwent scalp reconstructive surgery at a single center from 2010 to 2019. Patients data was collected from a medical database. Patients that underwent excisions of osteomas, lipomas, or epidermal cysts were excluded because there were no or minimal skin defects after surgery.

This study was reviewed and approved by the Institutional Review Board of Inje University Paik Hospital (IRB No. 202004-018) for human subject research and patient data was obtained by informed consent.

We examined selected patients through chart review, and collected the following data: defect etiology, location, size, depth, reconstructive procedure, postoperative complications, and whether the patient required revision surgery. Etiology was classified into postoperative alopecia, acute burn, malignant tumor resection, soft-tissue defect (after infection, operation, or trauma), and postradiation [4]. Defect location was classified into three categories: frontoparietal/vertex, temporal/temporoparietal, or occipital area. Defect size was classified into small $\left(<10 \mathrm{~cm}^{2}\right)$, medium $\left(10-50 \mathrm{~cm}^{2}\right)$, or large $\left(>50 \mathrm{~cm}^{2}\right)$ [1]. Defect depth was classified into soft-tissue defects without cranial bone exposure, and with cranial bone or dura mater exposure. Reconstructive procedures were categorized into primary closure, skin graft, local flap, pedicled regional flap, and free flap [1]. We also investigated whether a skin graft was performed for donor site coverage when a local or pedicled regional flap was performed.

We evaluated whether each of these factors had an impact on the choice of reconstruction procedure. With respect to the defect location, we evaluated whether a tight or loose scalp determined if primary closure was performed. The tight scalp is the region where the galea is located and is characterized by inelastic skin. We included the frontoparietal/vertex and occipital areas in this region for the purpose of this study [1]. The loose scalp is the region where the temporalis muscle and fascia are located and this region is characterized by more flexible skin. For this study, we included the temporal/temporoparietal area and lateral occipital areas in this region $[5,6]$. These three factors (defect location, size, and depth) were tested in order by the chi-square test, Spearman correlation test, and MannWhitney $U$ test.

Complications were divided into major or minor categories [3]. Major complications included flap necrosis, thrombosis, and skin graft necrosis that required revision surgery under general anesthesia. Additionally, cases that required revision surgery were further categorized by the reconstruction procedure and complications after the revision. Minor complications included flap congestion, partial skin graft loss, infection, hematoma, and dehiscence treated with only conservative therapy.

\section{RESULTS}

After applying the exclusion criteria, 94 patients were selected in total, and 98 cases of scalp reconstruction including revision surgery were performed. Among the 94 patients, 51 were male and 43 were female, while the mean age was $66.47 \pm 17.34$ years.

Table 1 shows the etiology of the scalp reconstructions. Malignant tumor resection (65.30\%) was the most common etiology, followed by posttraumatic soft-tissue defect (14.28\%), postoperative soft-tissue defect (9.18\%), postinfectious soft-tissue defect $(4.08 \%)$, burn $(3.06 \%)$, postoperative alopecia $(3.06 \%)$, and postradiation (1.02\%).

The factors affecting the choice of scalp reconstruction method are described in Table 2. The reconstruction of scalp defects was conducted in the frontoparietal/vertex and temporal/temporoparietal area in 45 cases, and in the occipital area in eight cases. The mean size of the scalp defects was $43.28 \pm 113 \mathrm{~cm}^{2}$, which was performed in 56 cases $(56.14 \%)$ of small size, 30 cases (30.61\%) of medium size, and 12 cases (12.24\%) of large size. In terms of the depth of scalp defects, there were 79 soft-tissue defects without cranial bone exposure, and 19 soft-tissue defects with cranial bone or dura mater exposure.

Tables 3 and 4 show the cases categorized by the characteristics of the defect and the type of scalp reconstruction method used and it also describes whether supportive surgery was per-

Table 1. Causes of scalp reconstruction $(n=98)$

\begin{tabular}{lc}
\hline Cause & No. of cases (\%) \\
\hline Malignant tumor resection & $64(65.30)$ \\
Soft-tissue defect & \\
Posttraumatic & $14(14.28)$ \\
Postoperative & $9(9.18)$ \\
Postinfectious & $4(4.08)$ \\
Burn & \\
Scalding & $3(3.06)$ \\
Radiation & $1(1.02)$ \\
Postoperative alopecia & $3(3.06)$ \\
\hline
\end{tabular}


formed simultaneously. There was a statistically significant difference $(p=0.011)$ between the proportion of primary closures performed versus no primary closure on loose scalps (51.11\%) and the proportion of primary closures performed versus no

Table 2. Defect classification and reconstructive procedures $(n=98)$

\begin{tabular}{lc}
\hline Variable & No. of cases (\%) \\
\hline Location & $45(45.91)$ \\
Frontoparietal/vertex & $45(45.91)$ \\
Temporal/temporoparietal & $8(8.16)$ \\
Occipital & \\
Size & $56(57.14)$ \\
Small $\left(<10 \mathrm{~cm}^{2}\right)$ & $30(30.61)$ \\
Medium $\left(10-50 \mathrm{~cm}^{2}\right)$ & $12(12.24)$ \\
Large $\left(>50 \mathrm{~cm}^{2}\right)$ & \\
Depth & $79(80.61)$ \\
Cranial bone exposure $(-)$ & $19(19.39)$ \\
Cranial bone or dura mater exposure (+) & \\
Reconstructive procedures & $36(36.73)$ \\
Direct closure & $27(27.55)$ \\
Skin graft & $10(10.2)$ \\
Local flap only & $7(7.14)$ \\
Local flap + skin graft & $2(2.04)$ \\
Regional flap only & $13(13.26)$ \\
Regional flap + skin graft & $3(3.06)$ \\
Free flap &
\end{tabular}

primary closure on tight scalps $(24.52 \%)$. Within the small size defect group, primary closures $(58.92 \%)$ were common, followed by local flap (19.64\%), skin graft (16.07\%) and regional flap (53.57\%). There were no free flap procedures performed in this group. Within the medium size defect group, skin grafts

Table 3. Reconstructive procedures depending on defect size and depth

\begin{tabular}{|c|c|c|c|c|c|c|}
\hline \multirow[b]{2}{*}{ Variable } & \multicolumn{5}{|c|}{ Reconstructive procedures } & \multirow[b]{2}{*}{$p$-value } \\
\hline & $\begin{array}{l}\text { Direct } \\
\text { closure }\end{array}$ & $\begin{array}{l}\text { Skin } \\
\text { graft }\end{array}$ & $\begin{array}{l}\text { Local } \\
\text { flap }\end{array}$ & $\begin{array}{l}\text { Regional } \\
\text { flap }\end{array}$ & $\begin{array}{l}\text { Free } \\
\text { flap }\end{array}$ & \\
\hline Size & & & & & & 0.000 \\
\hline Small $\left(<10 \mathrm{~cm}^{2}\right)$ & 33 & 9 & 11 & 3 & 0 & \\
\hline Medium $\left(10-50 \mathrm{~cm}^{2}\right)$ & 3 & 15 & 2 & 9 & 1 & \\
\hline Large $\left(>50 \mathrm{~cm}^{2}\right)$ & 0 & 3 & 4 & 3 & 2 & \\
\hline Depth & & & & & & 0.000 \\
\hline Cranial bone exposure $(-)$ & 36 & 26 & 11 & 6 & 0 & \\
\hline $\begin{array}{l}\text { Cranial bone or dura ma- } \\
\text { ter exposure }(+)\end{array}$ & 0 & 1 & 6 & 9 & 3 & \\
\hline
\end{tabular}

$\mathrm{a}=0.479$.

Table 4. Reconstructive procedures depending on location

\begin{tabular}{lccc}
\hline Variable & Primary closure & No primary closure & $p$-value \\
\hline Location & & & 0.011 \\
Tight scalp & 13 & 40 & \\
Loose scalp & 23 & 22 & \\
\hline
\end{tabular}

Tight scalp=frontoparietal/vertex+occipital area; loose scalp=temporal/temporoparietal area.
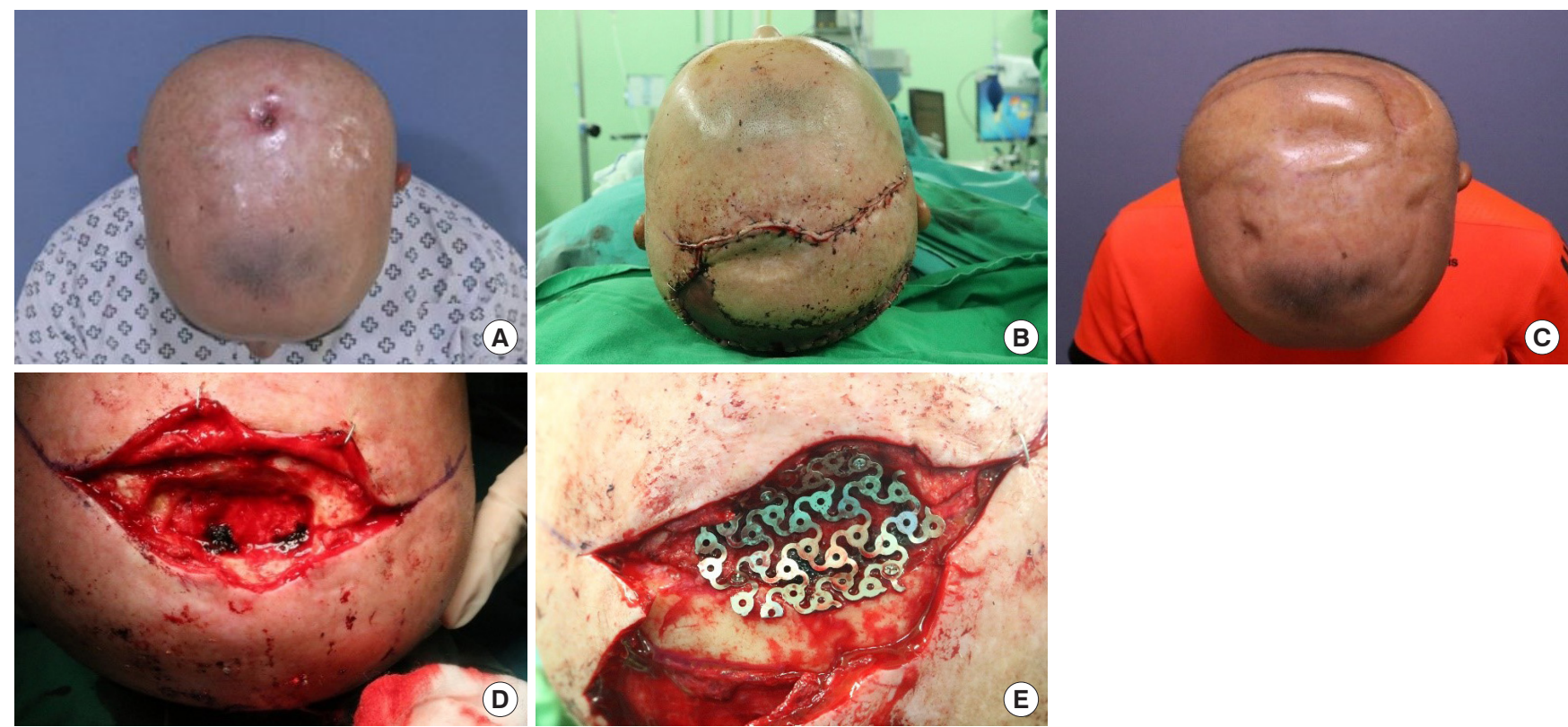

Fig. 1. A 39-year old man with scalp defect caused by radiation therapy. (A) Preoperative clinical photograph. (B) Intraoperative clinical photograph. (C) Postoperative clinical photograph. (D) Dura mater exposure and skull defect were observed. (E) Reconstruction with titanium mesh plate. Reconstruction of skin defect due to radiation therapy after a central neurocytoma operation. The bone defect was reconstructed with a titanium mesh plate. The superficial temporal artery-based regional flap was performed to cover the scalp defect and the donor site was covered with split-thickness skin graft. 

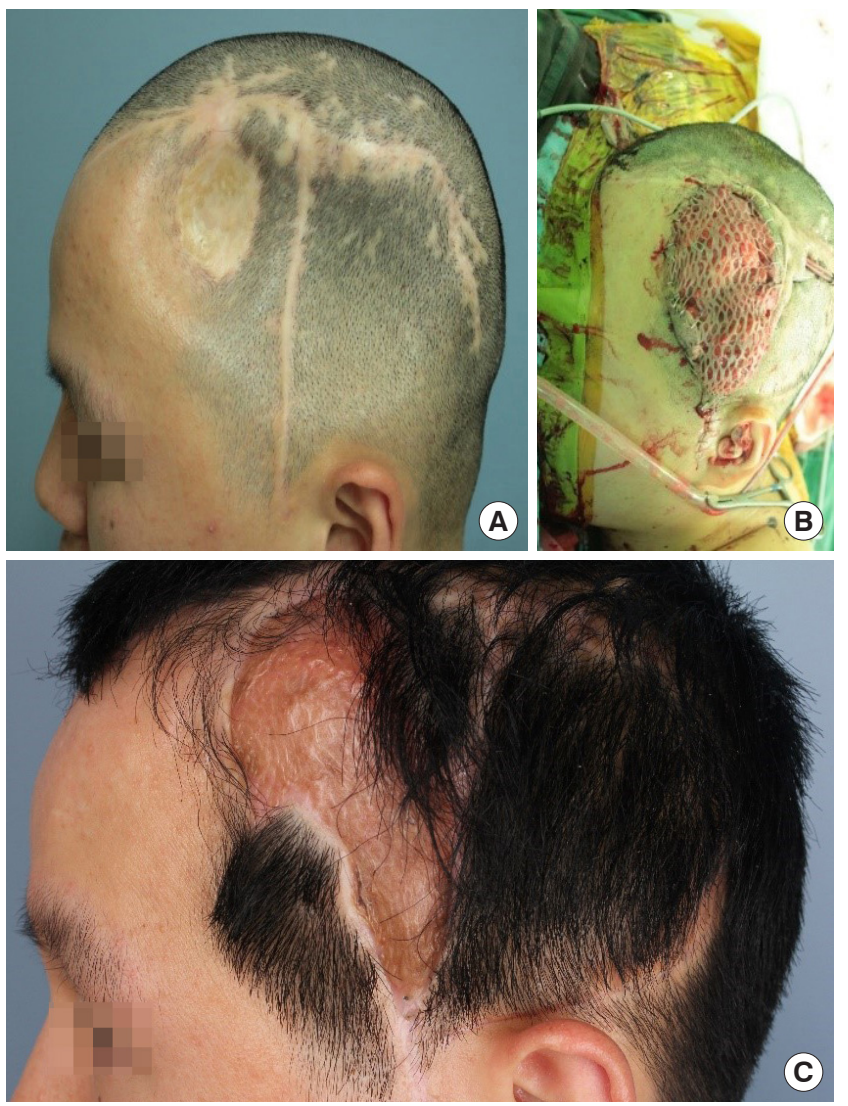

Fig. 2. Latissimus dorsi muscle free flap. (A) Preoperative clinical photograph. (B) Latissimus dorsi muscle free flap and skin graft. (C) Postoperative clinical photograph. Reconstruction of skull deformity after neurosurgery for traumatic epidural hemorrhage caused by a fall. Latissimus dorsi muscle free flap was performed with splitthickness skin graft.

(50\%) were most common, followed by regional flap (30\%), primary closure (10\%), local flap (6.66\%), and free flap (3.33\%). Within the large size defect group, local flap (33.33\%) was the most common procedure, followed by skin graft (25\%), regional flap (25\%) and free flap (16.66\%). There were no primary closures performed in this group. The choice of scalp reconstruction method was affected significantly by defect size $(\mathrm{R}=0.479, p<0.001)$. Additionally, this table shows the cases of scalp reconstruction depending on the scalp defect depth. Within the group of soft-tissue defects without cranial bone exposure, primary closure $(45.56 \%)$ was most common, followed by skin graft (32.91\%), local flap (13.92\%) and regional flap (7.59\%). There was no free flap performed in this group. Conversely, within the group of soft-tissue defects with cranial bone or dura mater exposure, regional flap (47.36\%) was mostly performed, followed by local flap (31.57\%), free flap (15.78\%), skin graft (5.26\%), and no primary closure. The choice of scalp reconstruction method has been affected significantly by the defect depth $(p<0.001)$. Fig. 1 shows a case where a superficial
Table 5. Complications of scalp reconstruction

\begin{tabular}{lc}
\hline Complications & No. of cases \\
\hline Major complications & 3 \\
Flap necrosis & 2 \\
Skin necrosis & \\
Minor complications & 1 \\
Flap congestion & 15 \\
Hematoma & 9 \\
Skin necrosis & 2 \\
Dehiscence & 2 \\
Infection & \\
\hline
\end{tabular}

temporal artery-based island flap procedure was performed on a patient with radiation therapy after a central neurocytoma operation. After a wide excision of the chronic ulcerative lesion, inflammation and necrosis of the bone was observed under the ulcerative lesion, and an ostectomy was performed with the aid of a neurosurgeon. The bone defect was reconstructed with a titanium mesh plate and the superficial temporal artery was checked by doppler ultrasonography. The regional flap procedure was performed to cover the scalp defect and the donor site was covered with split-thickness skin graft.

Fig. 2 shows the reconstruction of a skull deformity with a latissimus dorsi muscle free flap in a patient after neurosurgery for a traumatic epidural hemorrhage caused by a fall.

There were five major complications and 29 minor complications among the patients (Table 5). There were three cases of flap necrosis and two cases of skin necrosis within the major complications group. A total of four revision operations were performed, but revision surgery was not performed on one of the patients because the patient was transferred to another hospital. Hematoma (15 cases) was the most common minor complication, followed by skin necrosis (nine cases). These cases were managed conservatively and the wounds healed without further surgery. In the single case of flap congestion, leech therapy was applied for 1 week and the wound healed after 2 weeks of conservative treatment.

\section{DISCUSSION}

A deep understanding of scalp anatomy is essential in reconstructing scalp defects. Most of the scalp area has tight and inelastic properties, including the underlying galea and periosteum. When the scalp defect includes the tighter scalp, it is more difficult to perform a primary closure. This is a significant concern when choosing the best reconstruction procedure. Additionally, the surgeon must consider the defect size and depth, as well as prevention of alopecia, because the hair-bearing scalp 
affects the patient's general appearance. The choice of reconstruction method can vary significantly, as shown in our study. There are multiple methods utilized for the surgical treatment of scalp defects, with various surgical algorithms proposed [16]. To our knowledge, however, there is no established surgical treatment algorithm. This study established an algorithm by retrospectively studying scalp reconstruction performed in a single center for the last 10 years.

A primary closure is the first surgical method to consider for small size defects. If there is little tension at the time of primary closure after undermining the subcutaneous tissue around the defect, this can be considered as the first choice, because it reduces operation duration and minimizes alopecia [1]. One study obtained means of $83.3 \%$ and $92.2 \%$ tension reduction with $5 \mathrm{~cm}$ and $15 \mathrm{~cm}$ of undermining, respectively [7]. In our study, it was also observed that primary closure was more performed in small size defects less than $10 \mathrm{~cm}^{2}$ than in scalp defects greater than $10 \mathrm{~cm}^{2}$. In the loose scalp area, where primary closure was possible with relatively less tension after undermining, a primary closure was often performed significantly more than in the tight scalp. However, if primary closure is performed when the defect size is larger or the tension is great, the possibility of dehiscence of the surgical site or widening of the lineal alopecia cannot be excluded. In this case, other surgical treatments should be considered.

A skin graft usually should not be the first choice for scalp reconstructions, since it can cause poor cosmetic outcome such as alopecia, color mismatch, and height discrepancy. However, in some cases skin graft can be considered as an appropriate alternative if a well-vascularized tissue bed remains [8]. For example, in patients who are already bald, a flap operation such as a local flap, regional flap, or free flap, may not be appropriate because there would be no significant benefit and considering the possibility of flap necrosis, hematoma, and donor site morbidity. If the scalp defect is too large, the vascular condition of the patient is poor due to severe comorbidities such as old age, cardiovascular disease, or diabetes, or if the flap procedure threatens the life of the patient, a skin graft is an appropriate alternative to reduce invasiveness and duration of therapy.

In cases involving radiation therapy or bone exposure, other surgical procedures should be considered first because the healthy tissue is not suitable for skin grafting. However, a study showed that skin grafts with Integra may be a stable alternative for patients undergoing radiotherapy indicating the possibility of good results from skin grafts using bioengineered skin substitute [9].

A local flap is the most preferable surgical treatment for reconstructing scalp defects. A study found that a local flap is the most feasible method for reconstructing scalp defects less than $150 \mathrm{~cm}^{2}$ with a low complication rate (3.4\%) [1]. If the local flap can be performed on healthy tissue, it can be useful in compromised conditions such as with radiotherapy, infection, and postoperative skin necrosis [3]. Additionally, a local flap minimizes alopecia by covering defects using hair-bearing tissue and it provides "like with like" tissue, which can result in a favorable appearance by minimizing height discrepancy and color mismatch, even in patients without hair [1]. For a local flap to be successful, it is important to design one that is much larger than the defect size, and has a wide base, and to avoid suture lines in critical areas [10]. However, as the defect size increases, the likelihood that a skin graft on the donor site is the best procedure increases, and alopecia may be inevitable.

A regional flap is a surgery used to dissect the vascular pedicle and cover the scalp defect by using the vascularized flap. This operation is often used in large-size scalp defects. There are several types, including the lower island trapezius flap, latissimus dorsi musculocutaneous flap and the temporoparietal fascia flap [1]. The temporoparietal fascia flap, based on the superficial temporal artery, was used the most in our center. This flap is particularly useful for scalp defects in the frontal or temporal hairline regions because it can be designed with the hair-bearing scalp included [11]. Like a local flap, a regional flap can be performed in patients with compromised conditions, such as radiation therapy, infection, and postoperative skin necrosis [1]. In our study, regional flaps were performed more frequently in conjunction with a skin graft than were local flaps.

A free flap can be useful with medium or large size defects, especially in cases with previous radiation therapy, chronic infection, and neurocranial morbidity [1]. One study showed that a scalp free flap can survive postoperative radiation therapy after a malignant tumor resection and reconstruction surgery [12]. In another study, the free flap procedure was shown to be as safe and efficient in the elderly population as it is in other age groups, which means that age alone should not be considered a contraindication for a free flap [13]. A total of three free flap procedures (two latissimus dorsi muscle flaps and one anterolateral thigh flap) were performed in our study. A free flap allows for a more favorable cosmetic appearance by providing sufficient volume for the scalp deformity as the case of Fig. 2. A latissimus dorsi muscle free flap and skin graft were performed on this patient, thereby providing a favorable contour for the skull defects. The reason a latissimus dorsi myocutaneous free flap was not performed is that the subcutaneous fat on the latissimus dorsi muscle is too bulky, and therefore bulges after the surgery, creating poor skull contouring.

A tissue expander was not used in this center for several rea- 


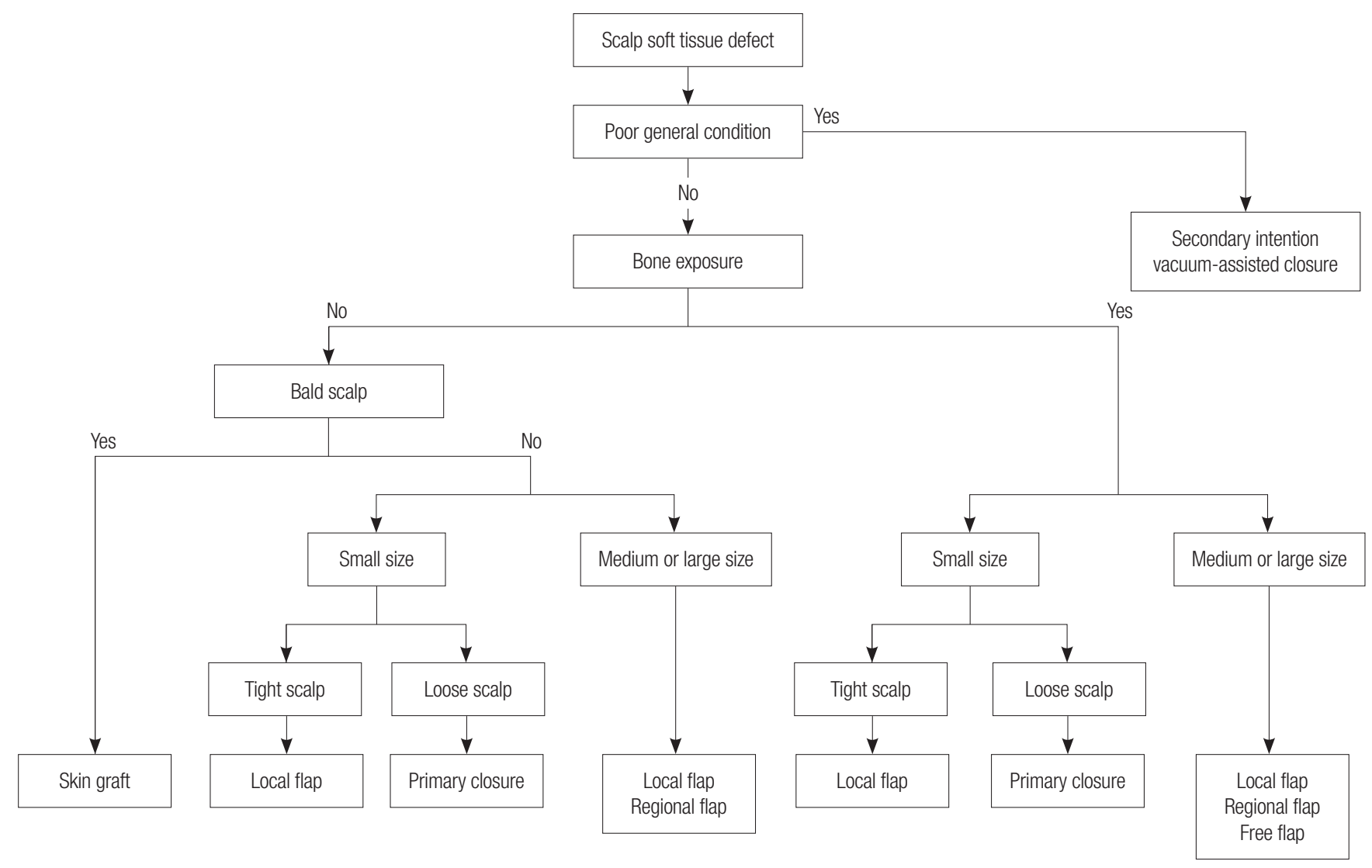

Fig. 3. Algorithm for reconstruction of scalp defect.

sons. First, due to the socioeconomic status of many of the patients that come to this center, patients generally refuse this option because of the high cost of the tissue expander coupled with the secondary surgery. Second, patients are understandably concerned with their appearance caused by the expander prior to the second surgery and, third, they do not want to be inconvenienced by repeatedly having to go to the hospital for inflation of the tissue expander. Most importantly, there is a risk of infection or inflammation due to the reaction to a foreign body, and this may ultimately necessitate removal of expander before performing the second surgery. Especially in patients with previous radiotherapy, chronic wound infection, or alloplastic material, tissue expanders should not be recommended due to the high complication rate $[1,12]$. Despite tissue expander insertion surgeries not being performed in this center, the procedure should be considered for patients with scalp alopecia acquired after other reconstruction surgeries.

To summarize, it is possible to select a reconstruction method for scalp defects based on the following algorithm (Fig. 3). First, conservative therapy such as a secondary intention or vacuumassisted closure should be considered when the patient's general condition is not good enough to perform reconstruction surgery under general anesthesia. If the patient's condition is good enough for a reconstruction, defect depth should be evaluated first. If cranial bone is not exposed at the defect but the patient is bald, a skin graft should be performed if primary closure is not possible. If the patient's scalp is hair-bearing, the size of the scalp defect should also be evaluated. In our study, the ratio of primary closure to no primary closure was significantly higher in defect sizes of less than $10 \mathrm{~cm}^{2}$ and on loose scalps than in the other categories. Therefore, if the scalp defect is on the loose scalp and the size is less than $10 \mathrm{~cm}^{2}$, primary closure should be considered the first choice. Even in the tight scalp, primary closure can be considered in small size defects where tension is not too tight to close the wound. However, if the tension is too great, a flap operation should be performed. Even if the defect size is small, it is critical to make the flap size large enough because, if it is not, there may be too much tension on the donor site, or a skin graft may need to be performed. In medium to large size scalp defects a primary closure is rarely performed, so, a local flap, regional flap, or free flap should be considered. In the case of a regional flap, a skin graft was more often accompanied than in a local flap, as mentioned above. In other words, alopecia is often involved after a regional flap so, if possible and even if the flap incision is large, a local flap should be considered first to cover the scalp defect. Finally, a reconstruction can 
be performed using a free flap when the local tissue is poor vascularity due to radiotherapy, or when a skull deformity is severe and a volume supplement is required.

There are some limitations in this study. First, this center did not perform reconstructions strictly following this algorithm. This was because we evaluated the data retrospectively and the operations were not performed by only one surgeon, meaning that the choice of reconstruction method was affected somewhat by the surgeon's preference. There were also considerations for patients with cardiovascular disease, diabetes mellitus, or other comorbidities as well as socioeconomic status that could not be factored into our study. Additionally, the number of patients in the large size defect group was much smaller than in the other defect size groups. Lastly, we should mention the case of skin graft for the reconstruction of dura mater-exposed scalp defect. We performed skin graft as temporary procedure for the protection of brain because dura mater was still exposed after craniectomy and duroplasty for skull fracture and traumatic epidural hemorrhage and patient condition was poor. Despite these limitations, this study may assist surgeons in their choice of reconstruction method before and during the surgery. In conclusion, multiple factors affect the choice of scalp reconstruction method. As there was no previously established algorithm approach for scalp reconstruction, we suggest that this algorithm, based on our 10 years of experience, will help surgeons better choose successful surgical managements for these patients.

\section{NOTES}

\section{Conflict of interest}

No potential conflict of interest relevant to this article was reported.

\section{Ethical approval}

The study was approved by the Institutional Review Board of Inje University Paik Hospital (IRB No. 2020-04-018) and performed in accordance with the principles of the Declaration of Helsinki. Written informed consents were obtained.

\section{Patient consent}

The patients provided written informed consent for the publication and the use of their images.

\section{ORCID}

Hyeon Uk Jang

https://orcid.org/0000-0003-1712-8839

Young Woong Choi

\section{REFERENCES}

1. Desai SC, Sand JP, Sharon JD, Branham G, Nussenbaum B. Scalp reconstruction: an algorithmic approach and systematic review. JAMA Facial Plast Surg 2015;17:56-66.

2. Leedy JE, Janis JE, Rohrich RJ. Reconstruction of acquired scalp defects: an algorithmic approach. Plast Reconstr Surg 2005;116:54e-72e.

3. Newman MI, Hanasono MM, Disa JJ, Cordeiro PG, Mehrara BJ. Scalp reconstruction: a 15-year experience. Ann Plast Surg 2004;52:501-6.

4. Steiner D, Hubertus A, Arkudas A, Taeger CD, Ludolph I, Boos AM, et al. Scalp reconstruction: a 10-year retrospective study. J Craniomaxillofac Surg 2017;45:319-24.

5. Temple CL, Ross DC. Scalp and forehead reconstruction. Clin Plast Surg 2005;32:377-90.

6. Brenner MJ. Scalp reconstruction. In: Branham GH, editor. Facial soft tissue reconstruction. Shelton: People's Medical Publishing House; 2011. p. 117.

7. Raposio E, Nordstrom RE, Santi PL. Undermining of the scalp: quantitative effects. Plast Reconstr Surg 1998;101:1218-22.

8. Worlicek C, Kaufmann R. Divided full-thickness skin graft for closure of circular and oval scalp defects. J Dtsch Dermatol Ges 2012;10:274-6.

9. Khan MA, Ali SN, Farid M, Pancholi M, Rayatt S, Yap LH. Use of dermal regeneration template (Integra) for reconstruction of full-thickness complex oncologic scalp defects. J Craniofac Surg 2010;21:905-9.

10. Mehrara BJ, Disa JJ, Pusic A. Scalp reconstruction. J Surg Oncol 2006;94:504-8.

11. Kim JC, Hadlock T, Varvares MA, Cheney ML. Hair-bearing temporoparietal fascial flap reconstruction of upper lip and scalp defects. Arch Facial Plast Surg 2001;3:170-7.

12. Hussussian CJ, Reece GP. Microsurgical scalp reconstruction in the patient with cancer. Plast Reconstr Surg 2002;109:1828-34.

13. Serletti JM, Higgins JP, Moran S, Orlando GS. Factors affecting outcome in free-tissue transfer in the elderly. Plast Reconstr Surg 2000;106:66-70. 